\title{
Fluctuating Mastectomy Rates Across Time and Geography
}

\author{
Carlos A. Garcia-Etienne, MD ${ }^{1,3}$, Mariano Tomatis, $\mathrm{MSc}^{2,3}$, Joerg Heil, $\mathrm{MD}^{4}$, Mahmoud Danaei, $\mathrm{MD}^{5}$, \\ Christoph J. Rageth, MD ${ }^{6}$, Lorenza Marotti, MSc ${ }^{7}$, Marco Rosselli del Turco, MD $^{7}$, \\ and Antonio Ponti, MD, MPH ${ }^{2,3}$
}

${ }^{1}$ Humanitas Cancer Center, Istituto Clinico Humanitas, IRCCS, Milan, Rozzano, Italy; ${ }^{2} \mathrm{CPO}$ Piemonte, AO Città della Salute e della Scienza di Torino, Turin, Italy; ${ }^{3}$ EUSOMA Data Centre, Turin, Italy; ${ }^{4}$ University Breast Unit Heidelberg, Heidelberg, Germany; ${ }^{5}$ Breast Unit, Aachen Marien Hospital, Aachen, Germany; ${ }^{6}$ Zurich Breast Center, Zurich, Switzerland; ${ }^{7}$ European Society of Breast Cancer Specialists (EUSOMA), Florence, Italy

\begin{abstract}
In 2009, 2 single-institution studies from the United States reported increasing mastectomy rates during the last decade. We have recently reported unilateral mastectomy trends from a European database and demonstrated a significant trend of decreasing mastectomy rates from $38.1 \%$ in 2005 to $13.1 \%$ in 2010 . A recent study from the SEER registry in the United States confirmed a previously reported decrease in mastectomy rates from $40.1 \%$ in year 2000 to $35.6 \%$ in 2005 , but showed a statistically significant increase in mastectomy rates up to $38.4 \%$ in 2008 . This report provides evidence that mastectomy trends may be in opposite directions in different geographical areas. The sharpest increase in mastectomy rates across all ages in the recent SEER study occurs right after year 2005, which interestingly corresponds with the time of publication of the meta-analysis by the EBCTCG that highlighted the importance of local control in breast cancer. The coincident timing raises the question of whether this evidence may have indirectly triggered an increase in mastectomy rates in the United States that would partially explain the observed trend, and more importantly, of whether an increase would be justified on this basis. Multiple factors influence the proportion between mastectomy and breast conservation, so it may be unreasonable to think of an optimal cutoff. There is not necessarily a right or wrong direction for mastectomy trends, but aiming to determine explanations for these differences may help
\end{abstract}

(C) Society of Surgical Oncology 2013

Published Online: 3 May 2013

C. A. Garcia-Etienne, MD

e-mail: carlos.garciaetienne@cancercenter.humanitas.it provide a clearer insight of the decision-making process involved in the surgical management of breast cancer.

A recent report from Mahmood and colleagues on national unilateral mastectomy rates for early-stage breast cancer in the United States brings back to light a discussion started in 2009 after the publication of 2 single-institution studies reporting increasing mastectomy rates in the last decade. ${ }^{1-3}$

The first study from the Mayo Clinic from a database of 5405 patients showed a significant decrease in mastectomy rates from $45 \%$ in 1997 to $31 \%$ in 2003, followed by a significant increase up to $45 \%$ in 2005 and $43 \%$ in $2006 .{ }^{2}$

The second single-institution study came from the Moffitt Cancer Center from a database of 5865 cases and showed a significant increase in mastectomy rates from $33 \%$ during the period from 1999 to 2003 to $44 \%$ between 2004 and 2007. ${ }^{3}$

Habermann et al. ${ }^{4}$ undertook a similar analysis based on the SEER registry with more than 230,000 cases with the aim to explore the national trend in the United States and showed that the proportion of women treated with mastectomy had a significant decrease from $41 \%$ in year 2000 to $37 \%$ in 2006. Interestingly, they pointed out a slight increase of $0.6 \%$ in mastectomy rates from 2005 to 2006 that was not statistically significant, and multivariate analysis showed that patients diagnosed in year 2006 were slightly more likely to undergo mastectomy than those diagnosed in 2005. Consequently, they acknowledged they could not exclude the possibility that findings from singleinstitution studies were reflecting a national trend in advance.

We have recently reported unilateral mastectomy trends from the eusomaDB, a central data warehouse of 
prospectively collected information from European Breast Units that have provided data according to the European Society of Breast Cancer Specialists (EUSOMA [originally European Society of Mastology]) requirements. 5,6 Our study included 15,369 cases with stage 0 -II breast cancer excluding pT3 lesions and demonstrated a significant trend of decreasing mastectomy rates from $38.1 \%$ in 2005 to $13.1 \%$ in 2010 . Our curve also suggested that mastectomy rates in the analyzed population were lower than those reported from studies in the United States. ${ }^{7}$

The updated report from Mahmood and colleagues on SEER data allows us to observe the continuation of the curve of Habermann's study until 2008. ${ }^{1,4}$ The more recent study included 256,081 women with T1-2 cases, whereas the previous one included cases with up to stage III disease ( $2 \%$ of invasive tumors had T3 lesions), but was mostly represented by early-stage cases as well (95\% stages $0-$ II). The updated curve confirms the decrease in mastectomy rates from $40.1 \%$ in year 2000 to $35.6 \%$ in 2005 and then it interestingly shows a sustained and statistically significant increase in mastectomy rates up to $38.4 \%$ in 2008. Additionally, multivariate analysis confirmed that women diagnosed in 2008 were more likely to undergo mastectomy than women diagnosed in 2005 . $^{1}$

Likewise, other recent report presented at the 2012 ASCO meeting analyzed mastectomy rates in cases with stage 0-II breast cancer at the MD Anderson Cancer Center (cohort of 8915 cases) and on the SEER registry (cohort of 359,572 cases). This report showed a significant decrease in mastectomies from $44.5 \%$ to $37.8 \%$ between 2000 and 2005 in the single-institution cohort and from $42.8 \%$ to $36.6 \%$ in the SEER cohort, followed by a significant increase in both curves with mastectomy performed in $48.6 \%$ of cases in the single-institution cohort and in $40.1 \%$ in the SEER cohort by $2008^{.}$

Conversely, another recent study from the SEER registry shows a decline in the use of mastectomy in favor of breast conservation. The study included 194, 860 patients with stage I disease diagnosed between 1998 and 2007. From these cases, 77,061 (40\%) underwent mastectomy and $117,799(60 \%)$ had a conservative procedure. Their multivariate analysis showed that patients diagnosed in the later years of the study were less likely to be treated with mastectomy (odds ratio [OR] 0.93, $95 \%$ CI 0.93-0.94, $p<.001)$. The rate of mastectomy continuously declined every 5-year interval, from $60 \%$ in 1988-1992, to $41 \%$ in 1993-1997, $33 \%$ in 1998-2002, and $32 \%$ in 2003-2007 $(p<.001)$. Nevertheless, the study included only 2 years (up to 2007) of the recent 3-year period reflecting increased mastectomy rates in Mahmood's report and year of diagnosis was grouped into 5-year intervals, failing to reflect yearly variations in mastectomy rates after 2005. Year clustering and patient population (stage I disease only) render its mastectomy rate curve unsuitable for comparison with those of previous reports. ${ }^{9}$

Given the differences in design, selection criteria, year of surgery, and availability of information from large databases, direct comparison of these studies may be inaccurate. However, these recent reports provide evidence that mastectomy trends may be in opposite directions in different geographical areas.

The majority of cases in our study derived from German Breast Units, so a wide representation of Europe is questionable. It would certainly be interesting to analyze these trends on a more widely represented European population, but data selection was conditioned by availability of uniform recent data for at least 5 consecutive years. It could also be argued that our curve depicts breast units that have undergone a process of certification to ensure a high level of quality care that may not represent the average European breast unit more suitable for comparison with the SEER registry curves. Still, it is in fact this process of certification that ensures the quality of data provided in our study.

Clinicopathologic characteristics such as young or older age, increase in tumor size, ductal carcinoma in situ, and lobular histology have been associated with increased probability of mastectomy across several studies (including ours). However, many other factors may impact the final surgical outcome in breast cancer such as use of preoperative breast MRI, patient awareness and concern about family history and hereditary breast cancer, increased patient education regarding reconstruction options, and the decision to undergo contralateral prophylactic mastectomy. Lack of information about these factors in the eusomaDB precluded an analysis in our original report.

From the population selected for our trend analysis, only $16 \%$ of mastectomy cases underwent immediate reconstruction and information about delayed reconstruction is not included in the database. It is not clear if immediate reconstruction is routinely offered in these centers or what factors influence these decisions. Recent data suggest that women are 4 times more likely to choose mastectomy rather than breast conservation when presented with options for breast reconstruction. ${ }^{10}$ This could be especially true in borderline candidates for breast conservation where availability of reconstruction may influence a shift toward mastectomy. In spite of that, recent studies about mastectomy rates do not include detailed information about postmastectomy reconstruction, hindering reliable observations on this issue.

It would be helpful to determine if there are differences among countries or centers on the extent of patient involvement for surgical decisions and the number and quality of available sources of information for patients such as printed materials provided by clinicians and online content presented in their own language. Yet, although these 
factors may be preoperative determinants of the type of surgery, they are not routinely documented prospectively.

Increase in mastectomy rates in patients younger than 40 years starts as early as 2003-2004 in Mahmood' s report. ${ }^{1}$ However, it is worth pointing out that the sharpest increase across all ages occurs right after year 2005, which interestingly corresponds with the time of publication of the meta-analysis by the Early Breast Cancer Trialists' Collaborative Group (EBCTCG) that ended the long-term debate on the importance of local control by showing that its improvement at 5 years resulted in a statistically significant improvement of both breast-cancer survival and overall survival at 15 years. ${ }^{11}$ The coincident timing raises the question of whether this evidence may have indirectly triggered an increase in mastectomy rates in the United States that would partially explain the observed trend, and more importantly, of whether an increase would be justified on this basis. If this conjecture is plausible, an essential question would then be why this evidence did not have the same impact on mastectomy rates of the eusomaDB? Our initial study suggested that, regardless of trend direction, mastectomy rates in the United States studies were higher compared with those observed in our database even in the lowest ranges reported. A sound explanation may be simply that the somehow lower threshold for mastectomy in U.S. centers was perhaps more susceptible to the information presented by the EBCTCG study and that the eusomaDB curve could be showing a less steep decrease in trend. Anyhow, this statement would remain hypothetical, as no study provides information to assess this appreciation.

The rates of local recurrence after breast-conserving surgery for early-stage cases originally reported in some seminal studies have decreased considerably. The 20-year recurrence rate in the ipsilateral breast in the NSABP B-06 trial started in 1976 was $14.3 \%$, whereas in the NSABP trials conducted in the 1990s, the latest reported 10-year local recurrence rates ranged from 3.5 to $6.5 \% .^{12,13}$ Absolute reductions in the rate of local recurrence would further decrease the likelihood of offering a survival benefit with more aggressive surgical procedures to current candidates for breast conservation.

An additional aspect that may impact the choice of surgical therapy accounting for differences in mastectomy rates across geography may be unique economic factors of each health system influencing availability of reconstruction or radiotherapy. Further studies would be needed to determine these differences.

The difficulty in determining what should be considered an adequate mastectomy rate lies on the multifactorial nature of an assessment process that includes not only important staging and clinicopathologic features but also aspects as personal preferences. Therefore, it may be unreasonable to think of an optimal fixed cutoff. The best proportion between mastectomy and breast conservation in clinical practice is ultimately defined by exercising a shared decision between the patient and the surgeon after having a thorough informed discussion. There is not necessarily a right or wrong direction for mastectomy trends, but aiming to determine explanations for these differences may help provide a clearer insight of the decision-making process involved in the surgical management of breast cancer.

\section{REFERENCES}

1. Mahmood U, Hanlon AL, Koshy M, Buras R, Chumsri S, Tkaczuk $\mathrm{KH}$, et al. Increasing national mastectomy rates for the treatment of early stage breast cancer. Ann Surg Oncol. 2012;20:1436-43.

2. Katipamula R, Degnim AC, Hoskin T, Boughey JC, Loprinzi C, Grant CS, et al. Trends in mastectomy rates at the Mayo Clinic Rochester: effect of surgical year and preoperative magnetic resonance imaging. J Clin Oncol. 2009;27:4082-8.

3. McGuire KP, Santillan AA, Kaur P, Meade T, Parbhoo J, Mathias $\mathrm{M}$, et al. Are mastectomies on the rise? A 13-year trend analysis of the selection of mastectomy versus breast conservation therapy in 5865 patients. Ann Surg Oncol. 2009;16:2682-90.

4. Habermann EB, Abbott A, Parsons HM, Virnig BA, Al-Refaie WB, Tuttle TM, et al. Are mastectomy rates really increasing in the United States? J Clin Oncol. 2010;28:3437-41.

5. European Society of Breast Cancer Specialists Database. http://www.eusomadb.org. Accessed March 2013.

6. EUSOMA: the requirements of a specialist Breast Unit. Available from: http://www.eusoma.org/doc/EusomaBURequirements2010. pdf. Accessed March 2013.

7. Garcia-Etienne CA, Tomatis M, Heil J, Friedrichs K, Kreienberg $\mathrm{R}$, Denk A, et al; eusomaDB Working Group. Mastectomy trends for early-stage breast cancer: A report from the EUSOMA multiinstitutional European database. Eur J Cancer. 2012;48:1947-56.

8. Yi M, Mittendorf EA, Hwang RF, Babiera G, Kuerer HM, Crow $\mathrm{J}$, Lange $\mathrm{R}$, et al. Are mastectomy rates really increasing? Experiences from a single institution and a population-based database. 2012 ASCO Annual Meeting. J Clin Oncol. 2012; 30(suppl; abstr 1028).

9. Showalter SL, Grover S, Sharma S, Lin L, Czerniecki BJ. Factors influencing surgical and adjuvant therapy in stage I breast cancer: a SEER 18 database analysis. Ann Surg Oncol. 2013;20:1287-94.

10. Alderman AK, Hawley ST, Waljee J, Mujahid M, Morrow M, Katz SJ. Understanding the impact of breast reconstruction on the surgical decision-making process for breast cancer. Cancer. 2008;112:489-94.

11. Early Breast Cancer Trialists' Collaborative Group (EBCTCG), Clarke M, Collins R, Darby S, McGale P, Correa C, et al. Effects of radiotherapy and of differences in the extent of surgery for early breast cancer on local recurrence and 15-year survival: an overview of the randomised trials. Lancet. 2005;366:2087-106.

12. Fisher B, Anderson S, Bryant J, Margolese RG, Deutsch M, Fisher ER, et al. Twenty-year follow-up of a randomized trial comparing total mastectomy, lumpectomy, and lumpectomy plus irradiation for the treatment of invasive breast cancer. $N$ Engl J Med. 2002;347:1233-41.

13. Anderson SJ, Wapnir I, Dignam JJ, Fisher B, Mamounas EP, Jeong JH, et al. Prognosis after ipsilateral breast tumor recurrence and locoregional recurrences in patients treated by breast-conserving therapy in five National Surgical Adjuvant Breast and Bowel Project protocols of node-negative breast cancer. J Clin Oncol. 2009;27:2466-73. 